\title{
Characterization of Coupled Transmission Lines Used in Integrated Circuit Packaging on Printed Circuit Boards
}

\author{
N. Tidjani, J. C. Le Bunetel, A. Ouchar, and Y. Raingeaud
}

\begin{abstract}
In this work, a characterization of coupled transmission lines used in integrated circuit packaging on printed circuit boards is presented. The method proposed is based on the method of moments and mathematical model based on analytical equations for fast extraction of parameters per unit length of coupled transmission lines lossless. The charge distribution is evaluated only once using the method of moments, in order to calculate per unit length parameters of capacity matrix in the presence of substrate. The elements per unit length of inductance matrix are then calculated by mathematical model based on analytical and empirical equations.
\end{abstract}

Index Terms-Method of moments, multiconductor lines parameters per unit length, packaging, $\mathrm{PCB}$.

\section{INTRODUCTION}

The industrial constraints evolution causes a break in the designing of printed circuit boards (PCBs). Today, the PCB must present a panel of analog and digital functions largest. It is necessary that all of these functions are integrated on smaller and smaller supports, and they are increasing speeds. However, this evaluation also leads to a degradation of signal quality and increase electromagnetic interference in the PCB cards. For that PCBs and their elements (edge connectors, microstrip lines), must be modeled using the transmission line methodology [1]-[4].

Several methods treated the transmission lines modeling by the parameters per unit length evaluation. Among these methods, the finite element method, invariance method, and method of moments (MoM) [5], [6].

In this article, a characterization of coupled transmission lines used in integrated circuit packaging on printed circuit boards is presented. We have chosen the method of moments and mathematical model based on empirical equations for fast characterization, and in order to simplify the extraction method for coupled microstrip transmission line. Matrix capacity $\mathrm{C}$ is computed only once in the presence of the substrate, by evaluating the corresponding charge distribution, using the method of images, the pulse basic function, and point matching of the method of MoM [7], [8]. The parameters per unit length of inductance matrix are then calculated analytically from a mathematical model in $M A T L A B$ routines based on analytical and empirical equations. For a coupled transmission lines characterization,

Manuscript received February 6, 2013; revised April 10, 2013. This work was supported in part by Campus France and GREMAN laboratory

Nassima Tidjani, Ali Ouchar are with University of Laghouat, Algeria (n.tidjani@mail.lagh-univ.dz).

Jean-Charles Le Bunetel, Yves Raingeaud are with University of François- Rabelais, Tours, France (lebunetel@univ-tours.fr) we have realized two PCBs, PCB I, and PCB II for one and two coupled transmission lines. Also PCB III is used with three coupled transmission lines. The measurements are performed by precision impedance analyzer 4294A, the calculations and simulations are done by Matlab routines and electromagnetic simulator Microwave office [9], [10].

\section{INTEGRAL EQUATIONS THEORY}

We consider a microstrip line of width $W$, which carrier a per unit length charge density $\rho_{1}$, assuming a zero- thickness strip, the potential can be written as follows [11], [12]:

$$
V(r)=-\frac{1}{2 \cdot \pi \cdot \varepsilon} \int_{0}^{W} \rho_{l}\left(r^{\prime}\right) \cdot \ln \left(\sqrt{\left(x-x^{\prime}\right)^{2}+y^{2}}\right) \cdot d x^{\prime}
$$

$r^{\prime}, r$ corresponds to the positions of source point, and observation point respectively. We divide the strip width $W$ of of $N$ sections of uniform width $\Delta$ "Fig.1", and we used the pulse basic function $P_{n}(x)$. The pulse basic function is defined unitary on the segment $\Delta$ and zero otherwise [4].

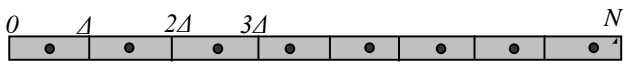

Fig. 1. Discretization of the strip into N segments.

The sum of the charges on the $N$ segments is equal to the total charge. $\alpha_{n}$ is the unknown charge density on a segment $n$ [13].

$$
\rho_{l}\left(r^{\prime}\right) \approx \sum_{n=1}^{N} \alpha_{n} P_{n}\left(r^{\prime}\right)
$$

When we replace "(2)" in "(1)", it will generate $N$ equations by evaluating "(3)" at $N$-point along the strip. It is segmented into $N$ computing the potential at the center of each segment. We assumed constant potential on each segment. $x_{m}$ is the position in the middle of segment $m$. For $m$ from 1 to $N$, we can write:

$$
V\left(x_{m}\right)=-\frac{1}{2 \pi \varepsilon} \sum_{n=1}^{N} \alpha_{n} \int_{x_{n-1}}^{x_{n}} P_{n}\left(x^{\prime}\right) \ln \left(\sqrt{\left(x_{m}-x^{\prime}\right)^{2}+y^{2}}\right) d x^{\prime}
$$

We introduce the boundary conditions $V(r)=V=1$. The final system of equations in matrix form is given by "(4)".

$$
[V]=[Z] \cdot[\alpha]
$$

With: 


$$
Z_{m n}=-\frac{1}{2 \pi \varepsilon} \int_{x_{n-1}}^{x_{n}} \ln \left(\sqrt{\left(x_{m}-x^{\prime}\right)^{2}+y^{2}}\right) d x^{\prime}
$$

We have for $m=n$ :

$$
\begin{aligned}
Z_{m n}= & \frac{\Delta}{2 \cdot \pi \cdot \varepsilon}[1-\ln (\Delta / 2)]+\frac{1}{4 \cdot \pi \cdot \varepsilon}\left\{\Delta \ln \left[(\Delta / 2)^{2}+4 d^{2}\right]\right. \\
& \left.-2 \Delta+8 d \tan ^{-1}\left(\frac{\Delta}{4 d}\right)\right\}
\end{aligned}
$$

And for $m \neq n$ :

$$
\begin{aligned}
Z_{m n}= & -\frac{\rho_{l}}{2 \cdot \pi \cdot \varepsilon}\left\{\left(\Delta_{m n}+\Delta / 2\right) \ln \left[\frac{\left(\Delta_{m n}+\Delta / 2\right)^{2}}{\left(\Delta_{m n}+\Delta / 2\right)^{2}+4 d^{2}}\right]-\left(\Delta_{m n}-\Delta / 2\right)\right. \\
& \left.\ln \left[\frac{\left(\Delta_{m n}-\Delta / 2\right)^{2}}{\left(\Delta_{m n}-\Delta / 2\right)^{2}+4 d^{2}}\right]-4 d\left[\tan ^{-1}\left(\frac{\Delta_{m n}+\Delta / 2}{2 d}\right)-\tan ^{-1}\left(\frac{\Delta_{m n}-\Delta / 2}{2 d}\right)\right]\right\}
\end{aligned}
$$

We set $B=Z^{1}$, we can find the total charge distribution $Q$

$$
Q=\left\{\sum_{\text {row } n} B_{i j}\right\}
$$

\section{PARAMETERS PER UNIT LENGTH}

At first we calculate the total charge distribution $Q$ with substrate by the method of moment. The method of moment divides each strip of width $W$ into $N$ uniform sections and uses the pulse basic function. Then the parameters per unit length of capacitance matrix $C$ are given for unitary voltage $V$ from “(1)".

$$
Q=C V
$$

We use the telegraph equation "(10)" for coupled transmission lines.

$$
\frac{\partial V(z, t)}{\partial z}=-\left[\begin{array}{ll}
L_{11} & L_{12} \\
L_{21} & L_{22}
\end{array}\right] \frac{\partial I(z, t)}{\partial t}
$$

where $L_{11}, L_{22}$ are the inductance of line 1 , line 2 respectively. $L_{12}, L_{21}$ are the mutual inductance between line 1 and line 2 . For two symmetrical microstrips we put:

$L_{12}=L_{21}=L_{\mathrm{m}}$, and $L_{11}=L_{22}=L$. The inductance matrix in equation "(10)" can be extended up to $\mathrm{N}$ transmission lines as follow [14]:

$$
L=\left[\begin{array}{ccccc}
L_{11} & L_{12} & \cdot & \cdot & L_{1 N} \\
L_{21} & L_{22} & \cdot & \cdot & \cdot \\
\cdot & \cdot & \cdot & \cdot & \cdot \\
\cdot & \cdot & \cdot & \cdot & \cdot \\
L_{N 1} & . & . & \cdot & L_{N N}
\end{array}\right]
$$

The parameters $L_{i i}$ of the matrix inductance depend only on the geometry of the lines, these elements can calculate from "(12)". The mutual parameters $L_{i j}$ are calculated from the empirical equation in "(13)". The valid ranges of the geometry parameters for the derived equation are summarized in Table I. With $H$ is the substrate height, $W$ the strip width, $T$ the strip height, $S$ the strips separation, $v_{0}$ the light speed, $\mu_{0}$ the vacuum permeability.

TABLE I: VALID RANGES OF THE GEOMETRY PARAMTERS

\begin{tabular}{cc}
\hline \hline$H$ & $0.1 \mathrm{~mm}<H<1.52 \mathrm{~mm}$ \\
$T$ & $17.2 \mathrm{~mm}<T<69.5 \mu \mathrm{m}$ \\
$S / H$ & $0.25<S / H<3.75$ \\
$W / H$ & $0.1<W / H<5$ \\
$S / W$ & $0.14<S / W<4$ \\
\hline
\end{tabular}

$$
\begin{aligned}
L_{i i}=\left\{\begin{array}{l}
\frac{60}{v_{0}} \ln \left[\frac{8 H}{W}+\frac{W}{4 H}\right] \\
\frac{120 \pi}{v_{0}} \ln \left[\frac{W}{H}+1.393+0.667 \ln \left(\frac{W}{H}+1.444\right)^{-1}\right] \frac{W}{H}>1
\end{array}\right. \\
L_{m}=\mu_{0}\left(\left[-0.415\left(\frac{H}{W}\right)^{-0.16}-2.38\left(\frac{T}{W}\right)^{1.18}\right]\left(\frac{S}{H}+1.07\right)^{-2.6}\right. \\
\left.+\left(\frac{S}{H}+0.89\right)^{-2.03}\left[0.418\left(\frac{H}{W}\right)^{0.13}+1.37\left(\frac{T}{W}\right)^{1.09}\right]\right)
\end{aligned}
$$

\section{VALIDATION AND MEASUREMENT}

We have realized a PCB I with one microstrip line deposited on the dielectric FR4, and PCB II contains coupled microstrip line. Also we have simulated PCB III with tree coupled microstrip.

The characteristics of PCB I, PCB II, and PCB III are summarised in Table II. The different measurement steps of parameters per unit length are summarized in figures (1-5). Fig. 2. and Fig. 3. illustrate the capacitance $C_{0}$, and inductance $L_{0}$ measurement, on PCB I

In Fig. 2. we put the impedance analyzer probe in the first connector with adapter, and we allow the second connector open. We find the capacitance expression $C_{0}$ by "(14)" in function of measure $C_{\text {meas }}$, connector capacitance $C_{\text {con }}$, and adapter capacitance $C_{\text {ada }}$.

$$
C_{0}=\left(C_{\text {meas }}-2 C_{c o n}-C_{a d a}\right) / l
$$

Also in Fig. 3, we put the probe in the first connector, and we allow the second connector in short circuit. The inductance expression $L_{0}$ is given by "(15)" in function of measure $L_{\text {meas }}$, and ground plane inductance $L_{\mathrm{GND}}$.

$$
L_{0}=\left(L_{\text {meas }}-L_{G N D}\right) / l
$$


TABLE II: DiMENSIONAL PARAMETERS OF TEST STRUCTURES

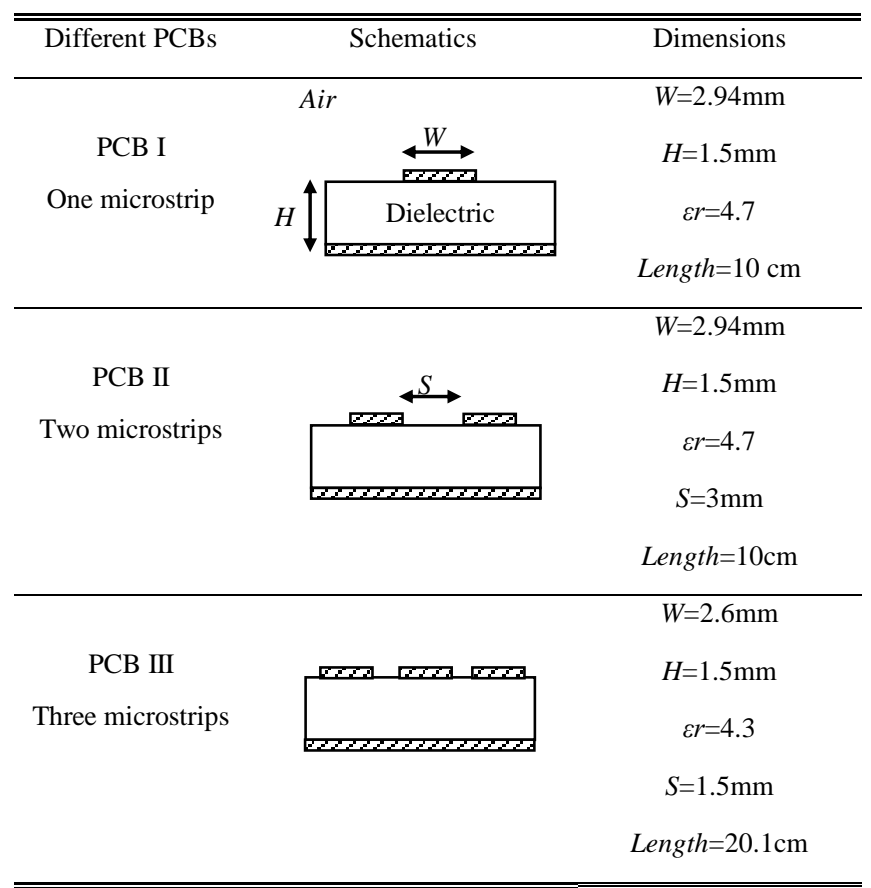

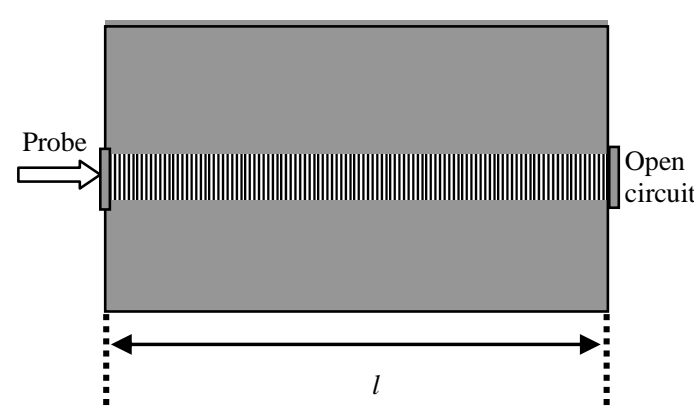

Fig. 2. Capacitance $\mathrm{C}_{0}$ measurement.

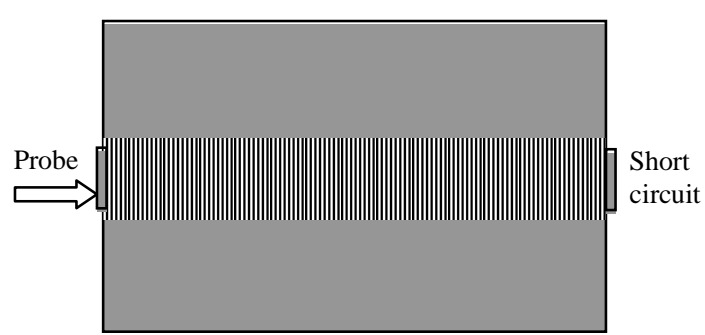

Fig. 3. Inductance $\mathrm{L}_{0}$ measurement.

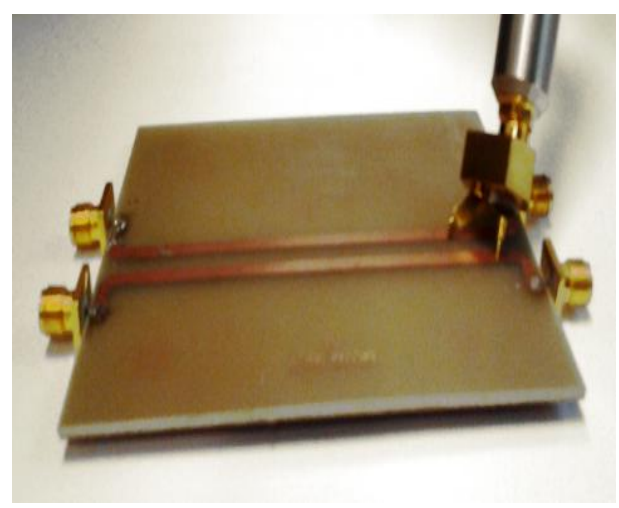

Fig. 4. Mutual capacitance $\mathrm{C}_{\mathrm{m}}$ measurement.

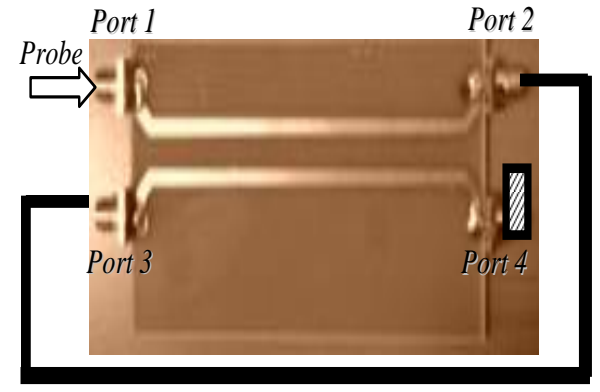

Fig. 5. Mutual inductance measurement $\mathrm{L}_{\text {meas } 1}$

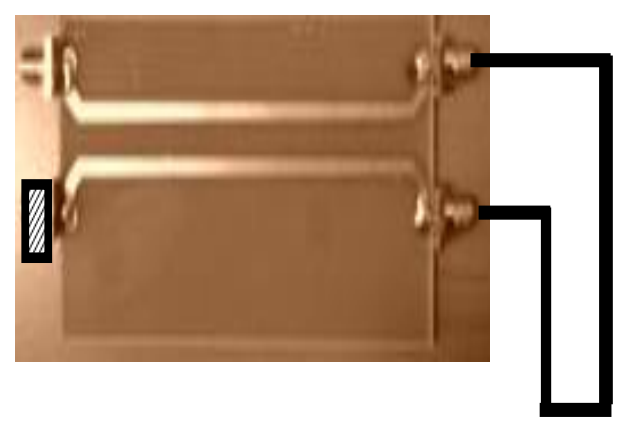

Fig. 6. Mutual inductance measurement $\mathrm{L}_{\text {meas2 }}$

For the mutual inductance measurement $\mathrm{L}_{\mathrm{m}}$ we have done two measures that are illustrated in Fig. 5. and Fig. 6. In Fig. 5. we put the probe in port 1 , connect port 2 and port 3 with a cable, and put port 4 in short circuit. The expression of measure is given by "(17)".

$$
L_{\text {meas } 1}=2 L-2 L_{m}+L_{G N D}+L_{\text {cable }}
$$

Also in Fig. 6. we put the probe in port 1, connect port 2 and port 4 with the same cable as the first measurement, and put port 3 in short circuit. The expression of measure is given by "(18)".

$$
L_{\text {meas } 2}=2 L+2 L_{m}+L_{G N D}+L_{\text {cable }}
$$

From these two measures we can find the expression of the mutual inductance $L_{m}$ by "(19)" in function of $L_{\text {meas } 1}$, and $L_{\text {meas } 2}$. The advantage of this method that the effects of the cable, ground plane, and strip are subtracted.

$$
L_{m}=\left(L_{\text {meas } 1}-L_{\text {meas } 2}\right) / l
$$

Finally, we can write all parameters per unit length of inductance and capacitance matrices in function of the done measurement, for symmetrical two coupled transmission lines by “(20)-(23)”.

$$
\begin{gathered}
L_{11}=L_{0} \\
L_{12}=L_{m} \\
C_{12}=C_{m} \\
C_{11}=C_{m}+C_{0}
\end{gathered}
$$

\section{RESULTS AND INTERPRETATION}

The Fig. 7. and Fig. 8. summarize the charge distribution, on the transmission line in PCB I depending by the position. We observe that the charge distribution has a low variation in 
the center, and augment at the edge of the strip, which is due to the edge effect. The Fig. 9. illustrates the capacitance convergence for PCB I, we observe when we increase the number of basic functions the precision increase. Table III illustrates the capacitance and inductance for PCB I.

A comparison of the results obtained by the proposed method, the MoM method, and measurement show a good agreement, with an error from $1.16 \%$ to $1.22 \%$. Table IV illustrates the capacitance and inductance matrices for PCB II. A comparison of the results obtained by the proposed method, the MoM method, and measurement shows a good agreement, with an error from $1.36 \%$ to $5.82 \%$.

Also, Table V illustrates the capacitance and inductance matrices for PCB III. A comparison of the results obtained by the proposed method, the MoM method, and simulation shows a good agreement [15]-[17].

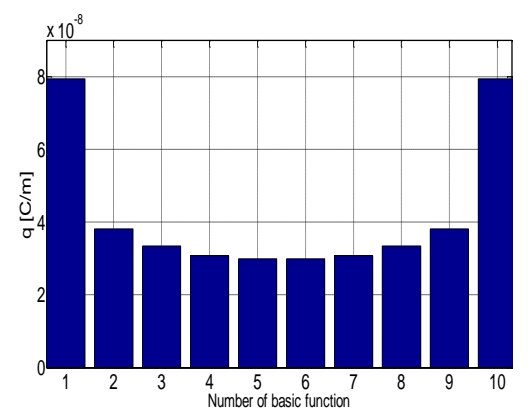

Fig. 7. Charge distribution for 10 divisions in PCB I.

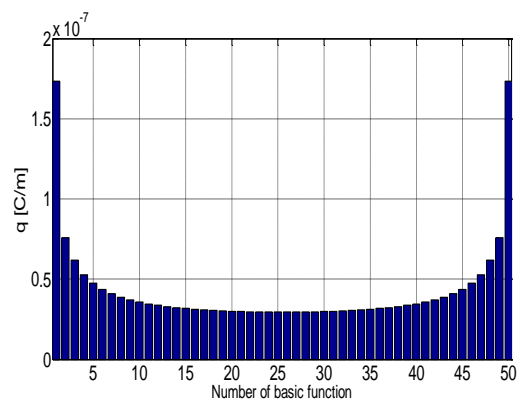

Fig. 8. Charge distribution for 50 divisions in PCB I.

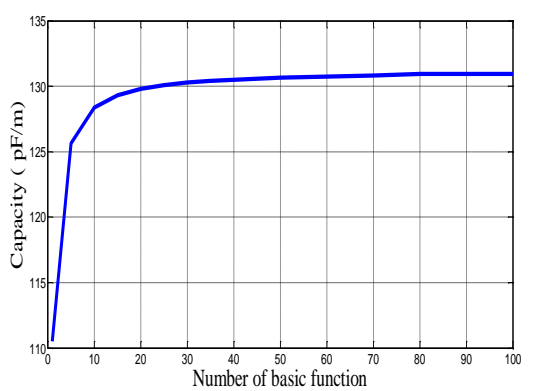

Fig. 9. Capacitance convergence for PCB I.

TABLE III: CAPACITANCE AND INDUCTANCE FOR PCB I

\begin{tabular}{cccc}
\hline \hline Parameters & Our work & MoM & Measure \\
\hline$L_{0}[\mathrm{nH} / \mathrm{m}]$ & 301.56 & 301.55 & 305.3 \\
$C_{0}[\mathrm{pF} / \mathrm{m}]$ & 130.6 & 130.65 & 129.1 \\
\hline \hline
\end{tabular}

TABLE IV: CAPACITANCE AND INDUCTANCE FOR PCB II

\begin{tabular}{cccc}
\hline \hline Parameters & Our work & MoM & Measure \\
\hline$L_{11}[\mathrm{nH} / \mathrm{m}]$ & 301.5 & 301.12 & 306.2 \\
$L_{12}[\mathrm{nH} / \mathrm{m}]$ & 26.83 & 26.304 & 27.2 \\
$L_{22}[\mathrm{nH} / \mathrm{m}]$ & 301.5 & 301.12 & 306.2 \\
$C_{11}[\mathrm{pF} / \mathrm{m}]$ & 130.81 & 130.8 & 138.9 \\
$C_{12}[\mathrm{pF} / \mathrm{m}]$ & 3.674 & 3.677 & 3.515 \\
$C_{22}[\mathrm{pF} / \mathrm{m}]$ & 130.81 & 130.8 & 129.1 \\
\hline \hline
\end{tabular}

TABLE V: CAPACITANCE AND INDUCTANCE FOR PCB III

\begin{tabular}{cccc}
\hline \hline Parameters & Our work & MoM & Simulation \\
\hline$L_{11}[\mathrm{nH} / \mathrm{m}]$ & 322.65 & 320.184 & 310.65 \\
$L_{12}[\mathrm{nH} / \mathrm{m}]$ & 53.48 & 51.94 & 53.18 \\
$L_{13}[\mathrm{nH} / \mathrm{m}]$ & 13.19 & 15.5 & 16.34 \\
$L_{22}[\mathrm{nH} / \mathrm{m}]$ & 322.65 & 317.94 & 307.5 \\
$L_{23}[\mathrm{nH} / \mathrm{m}]$ & 53.48 & 51.94 & 53.18 \\
$L_{33}[\mathrm{nH} / \mathrm{m}]$ & 322.65 & 320.18 & 310 \\
$C_{11}[\mathrm{pF} / \mathrm{m}]$ & 112.77 & 112.3 & 116.5 \\
$C_{12}[\mathrm{pF} / \mathrm{m}]$ & 9.303 & 9.306 & 10.305 \\
$C_{13}[\mathrm{pF} / \mathrm{m}]$ & 0.683 & 0.687 & 0.81 \\
$C_{22}[\mathrm{pF} / \mathrm{m}]$ & 113.98 & 113.5 & 117.9 \\
$C_{23}[\mathrm{pF} / \mathrm{m}]$ & 9.303 & 9.306 & 10.305 \\
$C_{33}[\mathrm{pF} / \mathrm{m}]$ & 112.77 & 112.3 & 116.5 \\
\hline \hline
\end{tabular}

\section{CONCLUSION}

In this work, we have chosen the method of moments and mathematical model, for fast characterization, and in order to simplify the extraction method for coupled microstrip transmission line. We have found a convergence of elements per unit length of matrix capacity acceptable in terms of time and memory calculation, which will be divided into two. The results are shown an acceptable agreement and faster between the proposed methods, the numerical method (MoM) and the measurement made. The knowledge of parameters per unit length of transmissions lines allows predicting crosstalk, and an eventual disruption that will microstrip or pines caused on neighbouring circuits and load.

\section{ACKNOWLEDGMENT}

The authors would like to thank Camps France, for their part to finance this project and M. Baudrier, physical measurement technician of the laboratory of GREMAN for his support in measurement.

\section{REFERENCES}

[1] A. Charoy, Comptabilité Electromagnétique, Fr: Dunod, 2012.

[2] S. Ghosh, and A. Chakrabarty, "Evaluation of per unit length parameters of multiconductor lines of high frequency integrated circuits by the method of rectangular subareas," in Proc. IMECS Conf., Hong Kong, 2008, pp. 1409.

[3] T. Heikkilä. (November 2007). On the Use time-domain methods to measure crosstalk systems, EETimes-Asia, [Online]. Available: 
http://www.eetasia.com/ARTICLES/2007NOV/PDF/EEOL_2007NO V16_TPA_EDA_TA.pdf?SOURCES=DOWNLOAD.

[4] T. Magesacher,"MTL-a multi wire transmission line modeling toolbox," International Journal of Computer and Electrical Engineering, vol. 5, no. 1, pp. 52-55, February 2013.

[5] S. Musa, M. Sadiku, and J. Clark, "Finite element analysis for electromagnetic parameters of multiconductor interconnects in multilayered dielectric media," International Journal of Advances in Applied Sciences, vol. 1, no. 4, pp. 181-190, December 2012.

[6] E. Metlevskis, "Analysis of rectangular microstrip structures by the method of moments," in Proc. of 18th International conference on microwave radar and wireless communications, Vilnius, 2010, pp. $1-4$.

[7] R. Clayton, Introduction to electromagnetic compatibility, 2nd edition, USA: John wiley \& Sons, 2006.

[8] W. Gibson, The Method of Moments in Electromagnetics, USA: Taylor \& Francis Group, 2006.

[9] Y. Sohn, J. Lee, H. Park, and S. Cho, "Empirical equations on electrical parameters of coupled microstrip lines for crosstalk estimation in printed circuit board," IEEE Trans on Advanced Packaging, vol. 24, no. 4, pp. 521-527, 2001.

[10] K. Soorya Krishna, S. Bhat, "Impedance matching in multi-layer interconnect structures to minimize signal reflections in high speed application," International Journal of Computer and Electrical Engineering, Vol. 4, No. 3, pp.345-349, June, 2012.

[11] F. Gardiol, Traité d'électricité: Electromagnétisme, Fr: Presses Polytechniques et Universitaires Romandes, 1996, vol. 3.

[12] Pérez, R. Carles, and R. Fleckinger, Électromagnétisme: Fondements et Applications, Fr: Dunod, 2001.

[13] N.Tidjani, J. Le Bunetel, and A. Ouchar, "Crosstalk analyses in coupled transmission lines used in telephony mobile," 17th IEEE Signal and Power Integrity, pp.155-158, Paris, 2013.

[14] R. Clayton, Analysis of Multiconductor Transmission Lines, USA: John Wiley \& Sons, IEEE Press, 2008.

[15] Microwave office getting started guide, AWR design environment, 2009.

[16] RF High Power Product Model Design Kits, AWR Corporation Microwave Office, 2011.

[17] I. Novak and B. Eged, "Measurement by vector network analyzer and simulation of crosstalk reduction on printed circuit boards with additional center traces," IEEE Instrumentation and Measurement Technology, pp. 269-274, Irvine, 1993.

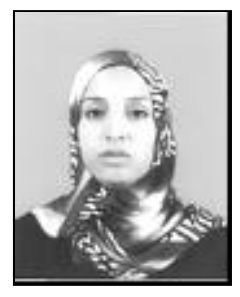

Nassima Tidjani received her Magisterium degree in electromagnetic compatibility in the electrical system from the university of Laghouat in Algeria in 2007, currently she is a PhD student in laboratory of GREMANat in university of Tours in France, her research interests are system modeling in EMC and Microwave, transmission lines, crosstalk, reduction method, at the GREMAN Laboratory, Tours, France.

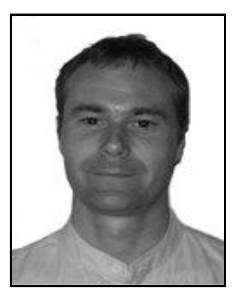

Jean-Charles Le Bunetel received the Ph.D. degree in electrical engineering from the University of Havre, LEPII laboratory, Le Havre, France, in 1997. He is currently Assistant Professor at the François Rabelais University, GREMAN, Tours, France. His research interests include power electronics, converter structures, EMI modeling, and associated EMC problems at the GREMAN Laboratory, Tours, France.

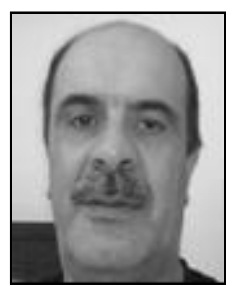

Ali Ouchar received the Ph.D. degree in telecommunication engineering from the Polytechnic of El Harach, Algiers, Algeria, in 2002. He is currently Professor at the university of Laghouat, Algeria. He is Director of the research laboratory LEDMASD in Laghouat in 2006. Member of the photonic network, and of renewable energy network Algiers. His research interests EMC problems, microwave devices, and fiber optics.

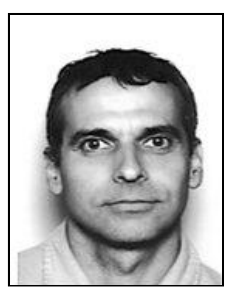

Yves Raingeaud received the Ph.D. degree in electrical engineering from the University of Limoges, IRCOM laboratory, Limoges, France, in 1993. He is currently Assistant Professor at the François Rabelais University, GREMAN, Tours, France. His research interests include power electronics switches, EMI modeling, and associated EMC problems at the GREMAN Laboratory, Tours, France. 